\title{
Bone marrow stem cells therapy alleviates vascular injury in a chronic obstructive pulmonary disease-obstructive sleep apnea overlap syndrome rat model
}

\author{
HONG BI ${ }^{*}$, JIAN HE* ${ }^{*}$ XU HE* ${ }^{*}$ JUNYI DU*, MIN CHEN, ZHAOMING HUANG, CHAO YANG, \\ LIJUAN YANG, HANG LI, KAIHUA ZHOU, QING WANG, LEWEI HE and ZHIXIAN JIN
}

Department of Pneumology, The First People's Hospital-Calmette Hospital of Kunming, Kunming, Yunnan 650224, P.R. China

Received June 1,2020; Accepted October 27, 2020

DOI: $10.3892 / \mathrm{mmr} .2020 .11707$

\begin{abstract}
Chronic obstructive pulmonary disease (COPD) and obstructive sleep apnea (OSA) are highly prevalent potential risk factors for systemic disease. Previous studies have reported that COPD and OSA are major independent risk factors for cardio- or cerebrovascular diseases. The present study aimed to investigate the role of bone marrow mesenchymal stem cells (BMSCs) on vascular injury in a COPD-OSA overlap syndrome (OS) rat model. Rats were randomly divided into three groups: Sham, OS model and BMSC. BMSC localization in major organs was detected via confocal laser fluorescence microscopy, and the aortic tissue pathological changes and related genes were measured using hematoxylin $\&$ eosin and Masson staining. Genes associated with vascular endothelial cell injury, including endothelin 1, vascular cell adhesion molecule 1 and endothelial nitric oxide synthase, were detected via reverse transcription-quantitative PCR and western blotting. Apoptosis of vascular endothelial cells was detected using TUNEL and immunofluorescence assays. The endothelial cell marker CD31 in injured vessels was analyzed via immunohistochemistry. BMSCs migrated into the heart, liver, spleen, lung, kidney, brain and aorta in the OS model. The green fluorescence expression of BMSCs demonstrated the highest level in the lung, followed by the aorta. Aortic tissue had a more severe vascular injury and increased apoptosis in the model group compared with the BMSC group. Vascular endothelial cell apoptosis was decreased in the BMSC group compared with the model group. The findings suggested that
\end{abstract}

Correspondence to: Professor Zhixian Jin, Department of Pneumology, The First People's Hospital-Calmette Hospital of Kunming, 1228 Beijing Road, Kunming, Yunnan 650224, P.R. China E-mail: jzx19706699@hotmail.com

*Contributed equally

Key words: bone marrow stem cells, aorta, apoptosis, endothelial, overlap syndrome, vascular injury
BMSCs could repair vascular injury by inhibiting endothelial cell damage and apoptosis. These data provide a theoretical basis for the treatment of cardiovascular diseases caused by OS with BMSCs.

\section{Introduction}

Chronic obstructive pulmonary disease (COPD) is a disease characterized by persistent airflow limitation (1). According to a report published by the World Health Organization, COPD will become the 4th leading cause of disease-related economic burden and the 3rd leading cause of mortality worldwide by 2030 (2). Obstructive sleep apnea (OSA) is a systemic disease with a high incidence and certain potential risks, such as cardiovascular and metabolic disease. Reports have indicated that OSA affects $17 \%$ of women and $34 \%$ of men in the US, and has a similar prevalence in other countries (3). Previous studies have reported that OSA is an independent risk factor for coronary heart disease, congestive heart failure and cerebrovascular disease $(4,5)$, although the correlation between these diseases and OSA has not been systematically studied.

David Flenley (6) was the first to create the term 'overlap syndrome' (OS), referring to the coexistence of COPD with OSA, and some studies have revealed a decreased survival rate among patients with OS compared with those with either COPD or OSA alone $(7,8)$. Clinical studies have reported that vascular endothelial injury occurs in both COPD and OSA, and endothelial dysfunction is more severe in patients with OS compared with patients with either condition alone and is correlated with the severity of the disease $(9,10)$. Intermittent hypoxia can induce the production of cytokines, including TNF- $\alpha$, IL- 6 and IL-8, and adhesion molecules by activating another important transcription factor, $\mathrm{NF}-\kappa \mathrm{B}$ to promote the development of inflammatory reactions and affect the formation of atherosclerosis $(11,12)$, thereby leading to vascular endothelial dysfunction (13). Therefore, identification of therapeutic strategies for vascular endothelial injury is crucial for the treatment of OS. To the best of our knowledge, research on vascular injury in OS is currently lacking. At present, there are few effective drugs in clinical treatment of OS (14). Therefore, it is important to conduct in-depth research on 
novel therapeutic measures to improve vascular endothelial injury and dysfunction.

Cell therapy has been used in the study of pulmonary diseases and the treatment of vascular complications (15). Previous studies have reported that embryonic stem cells and bone marrow (BM)-derived endothelial progenitor cells (EPCs) can differentiate into vascular endothelial cells (VECs) (16). COPD animal model studies have revealed that allogeneic EPCs transplanted into the trachea can prevent or delay disease progression by reducing inflammatory infiltration, relieving VEC apoptosis, increasing antioxidant activity and inhibiting proteolytic enzyme activity (17). BM mesenchymal stem cells (BMSCs) are considered cell transplantation and tissue engineering seed cells due to their convenience, simple separation, rapid expansion, high safety, low immunogenicity and multi-directional differentiation potential (18-20). BMSCs can quickly migrate from the BM into the peripheral circulation, mobilize to injury sites and differentiate into EPCs that further differentiate into VECs to repair the injury (21). MSCs have potential therapeutic roles in the treatment of obstructive sleep apnea-hypopnea syndrome (OSAHS) $(22)$ and COPD $(23,24)$. Previous studies have revealed that BMSCs can differentiate into alveolar epithelial cells in animals with lung injury and can repair pathological tissue $(25,26)$. However, to the best of our knowledge, few studies have been conducted to evaluate the effect of BMSCs on vascular injury in an OS rat model. Therefore, the aim of the present study was to investigate the role of BMSCs on vascular injury in COPD-OSA OS in vivo.

In the current study, an OS rat model was constructed using the smoke chamber exposure and intermittent hypoxia method, and then BMSCs were intravenously injected into rats. The localization of BMSCs was identified in some major organs using immunofluorescence assays, and the injured vascular tissues were collected to determine the damage via hematoxylin and eosin (H\&E) staining, reverse transcriptionquantitative (RT-q)PCR and western blot analysis. Apoptosis of VECs was detected using TUNEL and immunofluorescence assays. CD31 expression in injured vessels was detected via the immunohistochemical technique.

\section{Materials and methods}

Animal grouping. A total of 24 Sprague-Dawley rats (female; weight, $120 \pm 20 \mathrm{~g}$; age, 6 weeks) were purchased from Beijing Vital River Laboratory Animal Technology Co., Ltd. Rats were maintained in a clean-grade room under controlled temperature and lighting conditions with a 12-h light/dark photoperiod at $19-23^{\circ} \mathrm{C}$ and $40-60 \%$ humidity in the Animal Center of Kunming Medical University. The diet and drinking water of the rats were not restricted. In total, five rats were kept per cage. All animal experiments were approved by the Animals Ethics Committee of The First People's Hospital of Kunming and the Guide for the Care and Use of Laboratory Animals.

The OS rat model was established using the cigarette smoke and intermittent hypoxia exposure method according to our previous study (27). BMSCs were isolated from the femur and tibia of 1-week-old Sprague-Dawley rats (female; weight, $120 \pm 20 \mathrm{~g})$. The extraction method of BMSCs in rats were performed as described previously (27). A total of 24 female rats were randomly divided into three groups $(n=8)$ : Sham, OS model and BMSC. Rats in the sham group were treated with false smoke and air $\left(21 \% \mathrm{O}_{2}\right)$ exposure. The OS model was established using the cigarette smoke and intermittent hypoxia exposure method. The 6-week-old female SD rats were exposed to cigarette smoke every day at 8:00 to 8:30 a.m. and 5:30 to 6:00 p.m. (15 cigarettes at once, two times daily), intermittent hypoxia with $\sim 99 \%$ nitrogen $\left(\mathrm{N}_{2}\right)$ for $30 \mathrm{sec}$ and then exposed to air $\left(21 \% \mathrm{O}_{2}\right)$ every day for $90 \mathrm{sec}$ at 9:00 to 5:00 p.m. (30 times per hour, 8 h per day) for 8 weeks. All rats were successfully established as the OS model. BMSCs were labeled with the enhanced green fluorescent protein (GFP) lentivirus (cat. no. GM100202-2; Genomeditech Co., Ltd.) following the manufacturer's instructions. Following $24 \mathrm{~h}$ after cigarette smoke and intermittent hypoxia exposure, the rats in the model group were injected intravenously with PBS $(50 \mu 1)$, and those in the BMSC group were injected intravenously with $50 \mu \mathrm{l}$ BMSCs (total number of cells, 2x10 $/ \mathrm{rat}$ ) using a $1 \mathrm{ml}$ syringe via the tail vein (one time/week on the 7 th day of each week, for a total of four times). A total of 4 weeks after the last injection, the green fluorescence of BMSC in different tissues was observed under a confocal fluorescence microscope with magnification at x900 (Leica Microsystems $\mathrm{GmbH})$. Rats were euthanized with an overdose of anesthetic (sodium pentobarbital $150 \mathrm{mg} / \mathrm{kg}$; intraperitoneal). The rats had no heartbeat, continued involuntary breathing for 2-3 min and had no blink reflex, which was considered to indicate mortality. The descending aorta of aortic tissues were then collected from the rats.

The OS animal model was considered successfully established by evaluating lung morphologic analysis, as well as quantifying the emphysema and lung injury score, based on our previous study (27). Hypoxia and certain cardiovascular and cerebrovascular diseases can cause vascular injury in the large and medium-sized arteries (28), and so the most representative thoracic aorta (the descending aorta) was the primary research target. The descending aorta in the thoracic aorta is relatively thick and straight, and is easy to obtain sections from and to observe (28); thus, the descending aorta was used as the experimental tissue. In total, three of the OS model rats were injected intravenously with the GFP-labeled BMSCs (2x10\% rat) to investigate the location and differentiation of the transplanted BMSCs, and five of the OS model rats were injected intravenously with unlabeled BMSCs for examination with the TUNEL assay. All rats were used to perform H\&E staining and immunocytochemistry assays.

Aorta morphologic analysis. Aortic tissues were fixed in $4 \%$ formalin for $24 \mathrm{~h}$ at room temperature, embedded in paraffin, cut into $4-\mu \mathrm{m}$-thick sections and stained with $\mathrm{H} \& \mathrm{E}$ (cat. no. G1120; Beijing Solarbio Science \& Technology Co., Ltd.) for $10 \mathrm{~min}$ each at room temperature. The experiment was conducted according to the manufacturer's instructions. The tissue sections were examined via light microscopy at x400 magnification.

Masson staining. Aortic tissues were fixed in $4 \%$ formalin for $24 \mathrm{~h}$ at room temperature, embedded in paraffin, cut into 4- $\mu \mathrm{m}$-thick sections and stained with a Masson kit (cat. no. G1340; Beijing Solarbio Science \& Technology Co., 
Ltd.) for $5 \mathrm{~min}$ at room temperature. The experiment was conducted according to the manufacturer's instructions. In total, $\geq 3$ 200-fold fields of view were randomly selected for each slice in each group. The tissue sections were examined using light microscopy at x200 magnification. The collagen volume fraction (CVF) of the sections was determined using the ratio of pixel area of blue collagen fibers to total artery area using Image-Pro Plus 6.0 software (Media Cybernetics, Inc.).

TUNEL assay. The apoptosis of VECs was performed as previously described (27) using a TUNEL assay kit (cat. no. 11684817910; Roche Diagnostics, Inc.). Tissue samples were fixed in $4 \%$ formaldehyde for $24 \mathrm{~h}$ at room temperature and embedded in paraffin. Next, 4- $\mu$ m-thick paraffin sections were adhered to slides. Sections were deparaffinized by heating the slides for $30 \mathrm{~min}$ at $60^{\circ} \mathrm{C}$. The sections were washed with PBS twice. The sample was added to $50 \mu \mathrm{l}$ TUNEL assay solution and incubated at $37^{\circ} \mathrm{C}$ for $60 \mathrm{~min}$ in the dark. Then, the samples were washed with PBS twice. Sections were counterstained with hematoxylin for 5-10 min at room temperature to visualize nuclei. The film was sealed via anti-fluorescence quenching, and the sections were observed under a confocal microscope with x400 magnification. In total, three fields of view were randomly selected. The available excitation wavelength range was $450-500 \mathrm{~nm}$, and the emission wavelength range was 515-565 nm (green fluorescence). The tissue sections were examined via confocal microscopy.

Immunofluorescence. The tissue sections were placed in a $65^{\circ} \mathrm{C}$ incubator and baked for $15 \mathrm{~min}$. Paraffin sections were incubated at room temperature in xylene I for $15 \mathrm{~min}$, xylene II for $15 \mathrm{~min}$, ethanol I for $5 \mathrm{~min}$, anhydrous ethanol II for $5 \mathrm{~min}, 85 \%$ alcohol for $5 \mathrm{~min}, 75 \%$ alcohol for $5 \mathrm{~min}$ and distilled water to wash. Then, the samples were washed with $\mathrm{PBS}+0.1 \%$ Triton $\mathrm{X}-100 \mathrm{~T}$ (PBST) three times for 5 min each, at room temperature in a shaking bed. Tissue sections were placed in a repair box filled with EDTA antigen repair buffer, and antigen repair was conducted for $10 \mathrm{~min}$ at $100^{\circ} \mathrm{C}$ in the microwave oven. Then, slices were washed with $0.1 \%$ PBST three times, for 5 min each time, at room temperature in a shaking bed. The tissue was blocked with $5 \%$ v/v normal goat serum (cat. no. SL038; Beijing Solarbio Science \& Technology Co., Ltd.) for $30 \mathrm{~min}$ at room temperature. Sections were incubated with an anti-CD34 antibody (1:100; cat. no. A10796; ABclonal Biotech Co., Ltd.) overnight at $4^{\circ} \mathrm{C}$ and with a CoraLite594-conjugated secondary antibody (1:100; cat. no. SA00013-4; ProteinTech Group, Inc.) for $2 \mathrm{~h}$ at room temperature. Prior to DAPI staining of the nuclei, sections were washed three with PBS for 5 min each. Then, $10 \mu \mathrm{g} / \mathrm{ml}$ DAPI dye was added and incubated at room temperature in the dark for $10 \mathrm{~min}$. Film sealing was performed by washing with PBS three times. The section was sealed with anti-fluorescence quenching sealant. Sections were observed using a confocal microscope with x400 magnification.

Immunocytochemistry. Aortic tissues were fixed in $4 \%$ formalin for $24 \mathrm{~h}$ at room temperature, embedded in paraffin and cut into 4 - $\mu \mathrm{m}$-thick sections. An immunocytochemistry assay was performed according to a previous described method (27). The section was blocked with 5\% v/v normal goat serum (cat. no. SL038; Beijing Solarbio Science \& Technology Co., Ltd.) for $30 \mathrm{~min}$ at room temperature. Sections were incubated with an anti-CD31 antibody (1:100; cat. no. A3181; ABclonal Biotech Co., Ltd.) overnight at $4^{\circ} \mathrm{C}$ and then incubated with goat anti-rabbit $\operatorname{IgG}(\mathrm{H}+\mathrm{L})$ horseradish peroxidase (HRP)-conjugated secondary antibody (1:200; cat. no. AS014; ABclonal Biotech Co., Ltd.) for $2 \mathrm{~h}$ at room temperature. The reactions were visualized using a 3,3'-diaminobenzidine visualization kit (Fuzhou Maixin Biotech Co., Ltd.) for $10 \mathrm{~min}$ at room temperature. Sections were counterstained with hematoxylin to visualize nuclei, stained with hematoxylin for 5-10 $\mathrm{min}$ at room temperature and dehydrated. Sections were examined under a light microscope at a magnification of $\mathrm{x} 200$. Brown staining indicated immunoreactive cells, and blue staining indicated the nuclei. Sections were analyzed using Image-Pro Plus software (version 6.0; Media Cybernetics, Inc.) to obtain the integrated optical density.

$R T-q P C R$. Total RNA from aortic tissues was extracted using TRIzol $^{\circledR}$ (Takara Bio, Inc.). cDNA was synthesized from the RNA using a cDNA first strand synthesis kit (Takara Bio, Inc.) according to the manufacturer's instructions $\left(37^{\circ} \mathrm{C}\right.$ for $15 \mathrm{~min}$, $85^{\circ} \mathrm{C}$ for $5 \mathrm{sec}$ ). qPCR was performed using SYBR-Green PCR master mix (Takara Bio, Inc.). The qPCR reaction amplification system was as follows: Initial denaturation at $95^{\circ} \mathrm{C}$ for $10 \mathrm{~min}, 40$ of cycles of denaturation at $95^{\circ} \mathrm{C}$ for $15 \mathrm{sec}$, annealing at $60^{\circ} \mathrm{C}$ for $15 \mathrm{sec}$ and elongation at $60^{\circ} \mathrm{C}$ for $60 \mathrm{sec}$. The reaction was performed in a PCR amplification system (ABI 7300; Thermo Fisher Scientific, Inc.). The following primers were used: Endothelin-1 (ET-1) forward (F), 5'-ACC ACAGACCAAGGGAACAG-3' and reverse (R), 5'-GGTCTT GATGCTGTTGCTGA-3'; vascular cell adhesion molecule-1 (VCAM-1) F, 5'-TGACATCTCCCCTGGATCTC-3' and R, 5'-CTCCAGTTTCCTTCGCTGAC-3'; endothelial nitric oxide synthase (eNOS) F, 5'-TGACCCTCACCGATACAACA-3' and R, 5'-CTGGCCTTCTGCTCATTTTC-3'; and GAPDH F, 5'-CTCATGACCACAGTCCATGC-3' and R, 5'-TTCAGC TCTGGGATGACCTT-3'. Relative gene expression data was analyzed via the $2^{-\Delta \Delta \mathrm{Cq}}$ method (29).

Western blot analysis. Aortic tissues from all rats were extracted with RIPA lysis buffer (Beyotime Institute of Biotechnology). Protein concentration was determined using the BCA Protein Assay kit (Beyotime Institute of Biotechnology), and $35 \mu \mathrm{g}$ protein was analyzed with $10 \%$ SDS-PAGE. Then, the protein was transferred to the PVDF membrane. The membrane was treated in a blocking solution (5\% skimmed milk) at room temperature for $2 \mathrm{~h}$ and incubated with an anti-ET-1 (1:1,000; cat. no. A0686; ABclonal Biotech Co., Ltd.), anti-VCAM-1 (1:1,000; cat. no. A11236; ABclonal Biotech Co., Ltd.), anti-eNOS (1:1,000; cat. no. A1548; ABclonal Biotech Co., Ltd.), anti-CD31 (1:1,000; cat. no. A3181; ABclonal Biotech Co., Ltd.), anti-GFP Tag (1:1,000; cat. no. 50430-2-AP; ProteinTech Group, Inc.) or anti- $\beta$-actin antibodies (1:5,000; cat. no. 20536-1-AP; ProteinTech Group, Inc.) at $4^{\circ} \mathrm{C}$ for overnight, followed by incubation with HRP Goat Anti-Rabbit IgG $(\mathrm{H}+\mathrm{L})$ secondary antibody (1:5,000; cat. no. AS014; ABclonal Biotech Co., Ltd.) at room temperature for $1 \mathrm{~h}$. The membrane was detected with the ECL 
A
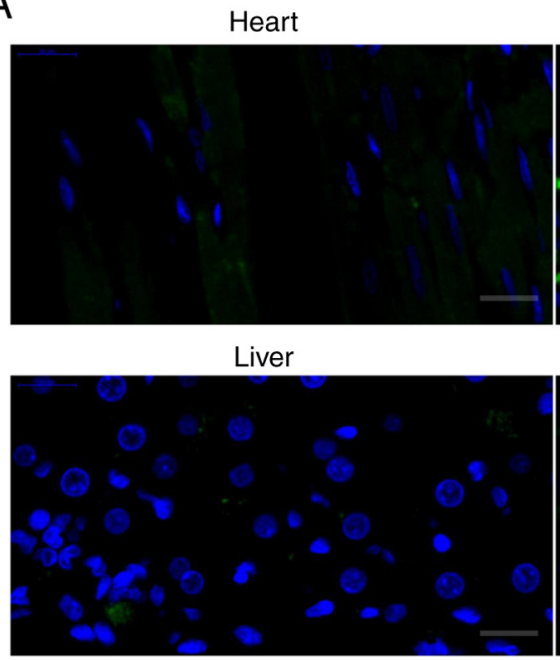

Spleen

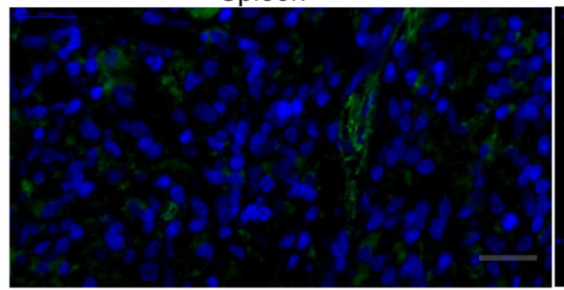

Lung

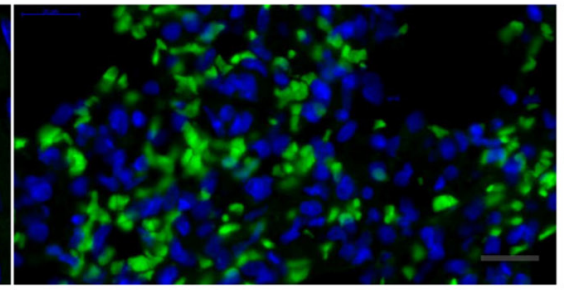

Kidney

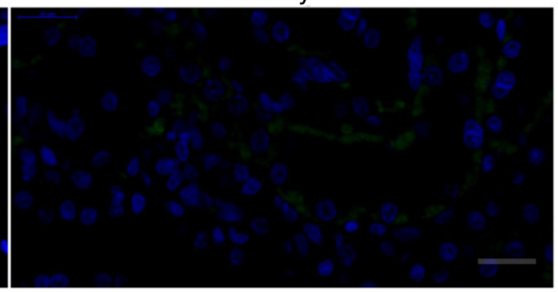

Brain

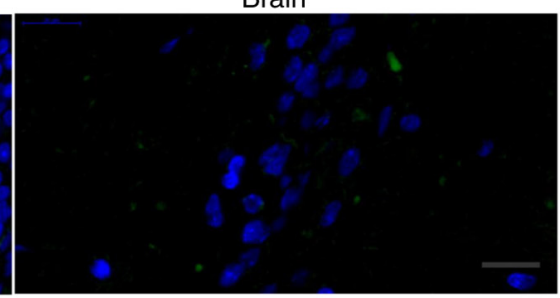

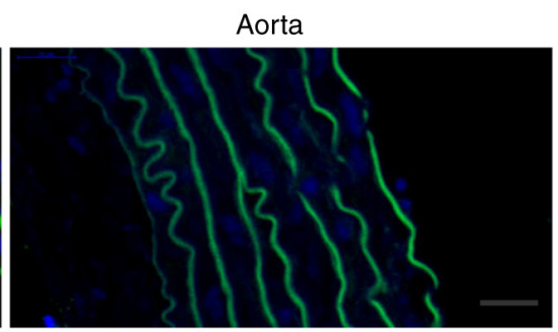

B

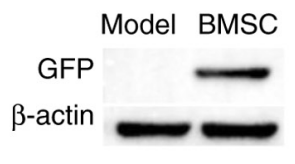

C

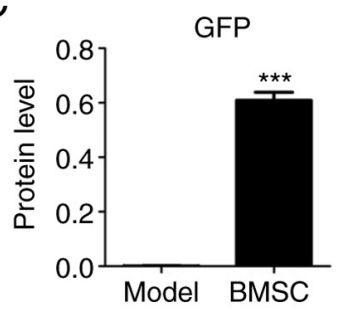

Figure 1. Localization of BMSCs in major organs of OS rats. (A) BMSC expression in the heart, liver, spleen, lung, kidney, brain and aortic tissues under a fluorescence microscope (magnification, x900). Scale bar, $20 \mu \mathrm{m}$. The nucleus is stained with DAPI. (B) Protein expression levels of GFP in the aortas were detected via western blot analysis. (C) Semi-quantitative results of western blotting in all groups. ${ }^{* * *} \mathrm{P}<0.001$ vs. the model group. GFP, green fluorescent protein; BMSC, bone marrow mesenchymal stem cells.

Detection reagents (EMD Millipore). The optical density of the resulting bands was determined using ImageJ 2x software (Rawak Software, Inc.), and the densitometry measurements were normalized to that of $\beta$-actin.

Statistical analysis. Data are presented as the mean \pm SD. Comparisons were assessed with one-way ANOVA followed by Tukey's post hoc test, using GraphPad Prism software version 5.0a (GraphPad Software, Inc.). All experiments were conducted $\geq 3$ times. $\mathrm{P}<0.05$ was considered to indicate a statistically significant difference.

\section{Results}

Body observation of rats in each group. All rats survived. In the OS group rats, the initial reaction to smoke was irritability, and then the rats exercised less and closed their eyes. When exposure to smoke was prolonged, rats gradually appeared to breathe abnormally and salivate, as well as had an intermittent cough. After 2-3 weeks of audible wheezing, their skin became yellow and signs of depression were observed, with a loss of appetite and a loss of weight. The rats in the BMSC group demonstrated normal activity, yellowing fur, a slightly decreased appetite and little change in body size. The rats in the sham group demonstrated normal activity before and after the experiment, good appetite, fat bodies, no shedding of fur and no cyanosis caused by breathing air (data not shown). The weight of rats was significantly decreased in the model group $(217.23 \pm 7.19 \mathrm{~g})$ compared with that in the sham group
(254.26 $\pm 9.92 \mathrm{~g})$, but no difference between the sham group and the BMSC group was observed $(251.78 \pm 6.34 \mathrm{~g})$.

Localization of BMSCs in OS rats. To investigate the localization of BMSCs in OS rats, GFP-BMSCs were injected into OS rats via the tail vein. As presented in Fig. 1, green fluorescence was identified in seven major organs of OS rats using confocal microscopy. It was found that green fluorescence was expressed in all seven tissues type, but was most obvious in lung and arterial tissues (Fig. 1A). This finding suggested that lung and arterial tissues may be severely damaged in the OS model, and that BMSCs could migrate to the damaged tissues. The GFP-labeled BMSCs were verified using western blot analysis. GFP protein expression was significantly higher in the BMSC group compared with that in the model group (Fig. 1B and C).

BMSCs repair arterial injury. The pathological morphology of arterial tissues was detected using H\&E staining. In the sham group, the vascular wall structure of the aorta was clear, and the boundary of the outer membrane, middle membrane and intima was clear (Fig. 2A). In the model group, the vascular wall structure of the aorta was disordered, and the boundary between the outer membrane, middle membrane and intima was not clear. Moreover, the elastic membrane of the media was not visible, and smooth muscle cells were disordered and swollen. Hyperplasia, cell morphology changes and endothelial swelling were also observed. In the BMSC group, the vascular wall structure of the aorta was 

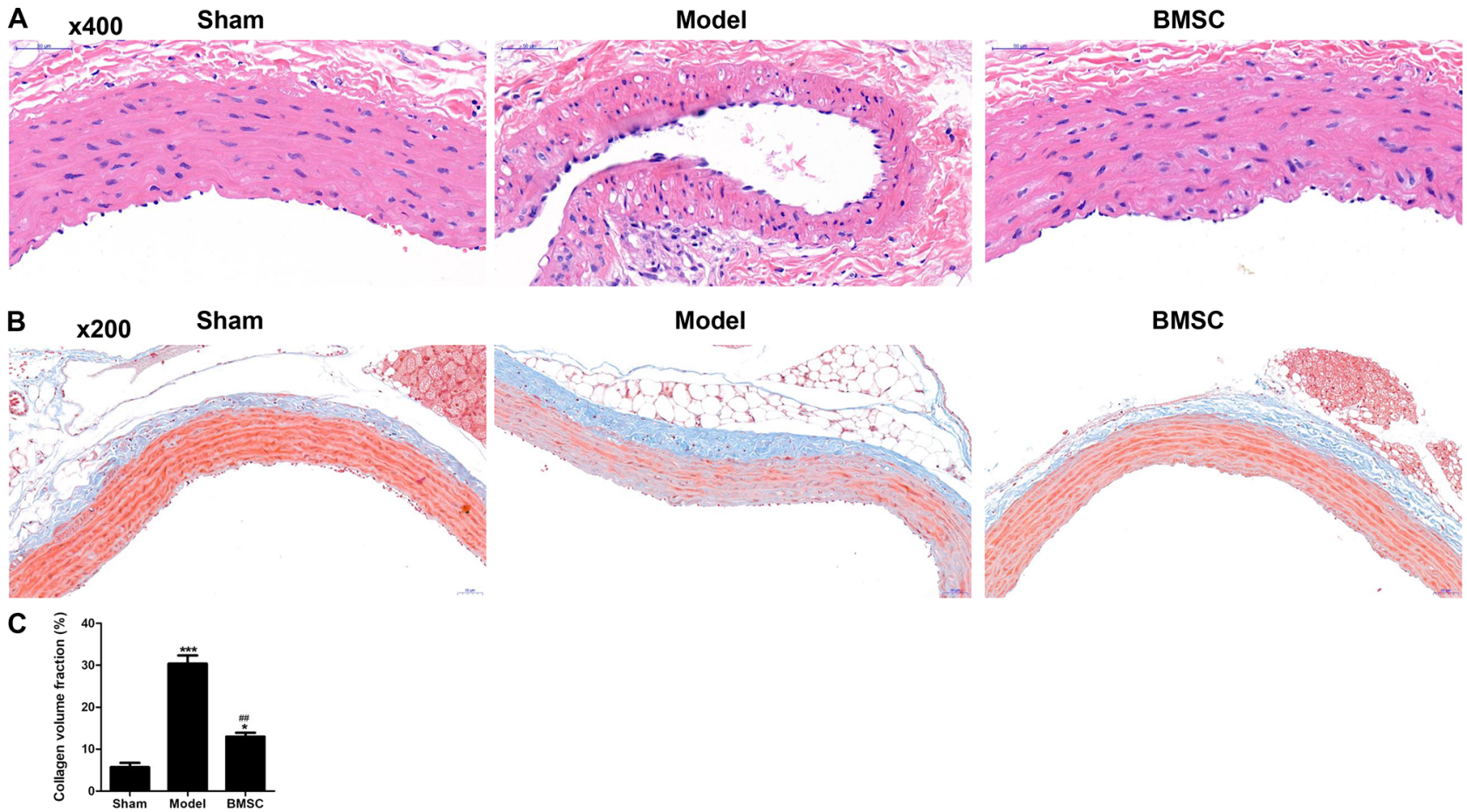

Model

BMSC

Figure 2. BMSCs inhibit the aortic injury induced by the OS model. (A) Hematoxylin and eosin staining of aortic tissue sections in the sham group, model group and BMSC group. Magnification, x400. (B) Collagenous fiber formation of vessels were detected via Masson staining. Magnification, x200. (C) Collagenous fiber was quantified using the collagen volume fraction. ${ }^{*} \mathrm{P}<0.05$ and ${ }^{* * * *} \mathrm{P}<0.001$ vs. the sham group; ${ }^{\# \#} \mathrm{P}<0.01$ vs. the model group. BMSC, bone marrow mesenchymal stem cells.

clear, the tunica media was thickened, the smooth muscle cells were proliferated, the arrangement of the vascular wall structure was not orderly and endothelial cells were slightly swollen (Fig. 2A). These results indicated that arterial tissues were severely damaged in the OS model, and that BMSCs can inhibit these damages.

BMSCs inhibit collagenous fiber formation in OS rats. The collagenous fibers of arterial tissues were examined using Masson staining. Compared with that of the sham group, the expression of collagen fibers (blue) in the outer and medial membrane of aortic tissues of the model group was markedly increased, and its elastic fibers (red) were notably decreased (Fig. 2B). Compared with that of the model group, the expression of collagen fibers in the BMSC group was significantly decreased, but was still higher compared with that in the sham group. The collagen fibers (blue) were also quantified based on the CVF (Fig. 2C). These results suggested that BMSCs repaired arterial injury by inhibiting the formation of collagen fibers.

BMSCs suppresses endotheliocyte injury in OS rats. To demonstrate whether BMSCs can suppress endotheliocyte injury, the endotheliocyte injury-related genes ET-1, VCAM-1 and eNOS were assessed via RT-qPCR and western blotting. The expression levels of ET-1 and VCAM-1 were significantly increased in the model group compared with the sham group, but were decreased in the BMSC group compared with the model group (Fig. 3A). The expression of eNOS was significantly decreased in the model group compared with the sham group, and was upregulated in the BMSC group compared with the model group. The results of the western blot analysis were consistent with the RT-qPCR results (Fig. 3B and C). These findings suggested that BMSCs repaired arterial injury by inhibiting endotheliocyte injury.

BMSCs suppress endotheliocyte apoptosis in OS rats. A TUNEL assay was performed to detect endotheliocyte apoptosis. The apoptotic cells under fluorescence microscopy displayed green fluorescence, while the endotheliocytes under fluorescence microscopy fluoresced red. The co-expression of red and green fluorescence indicated apoptotic endotheliocytes. Compared with the sham group, higher levels of endotheliocyte apoptosis were observed in the model group. Endotheliocyte apoptosis was significantly decreased in the BMSC group compared with the model group, although it was greater compared with that in the sham group (Fig. 4A and B). Quantitative analysis of the co-expression of green and red fluorescence indicated that BMSCs significantly suppressed the apoptosis of endotheliocytes induced by OS model (Fig. 4B). These results demonstrated that BMSCs repaired arterial injury by inhibiting endotheliocyte apoptosis.

BMSCs increase the expression of CD31 in aortic tissues of $O S$ rats. The expression of CD31 protein was detected using an immunohistochemical assay. Lower levels of brown staining were observed in the aortic tissues of the model group compared with that in the sham group (Fig. 5A and B). However, BMSC transplantation significantly increased the brown staining in the aortic tissues of OS rats (Fig. 5A and B). The quantitative results of the immunohistochemical assay 
A

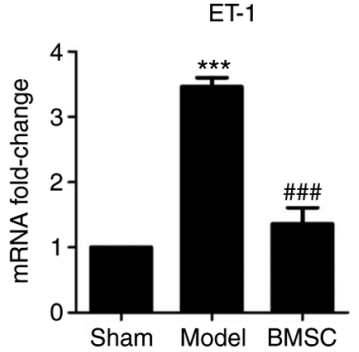

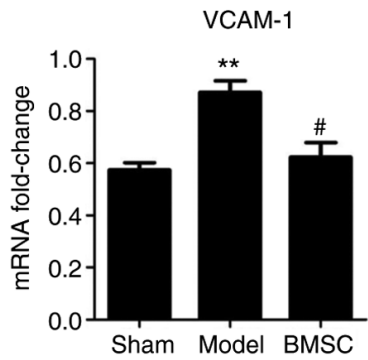

eNOS

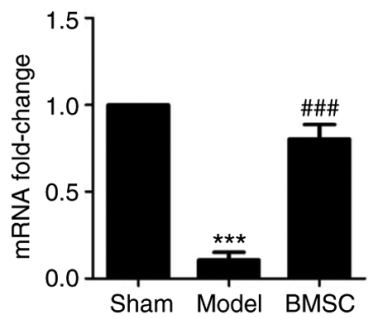

B

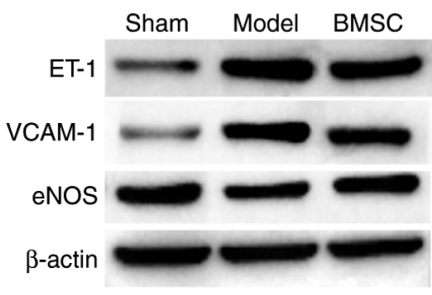

C

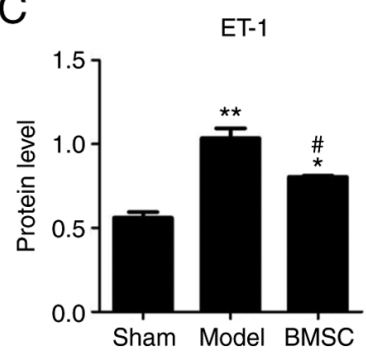

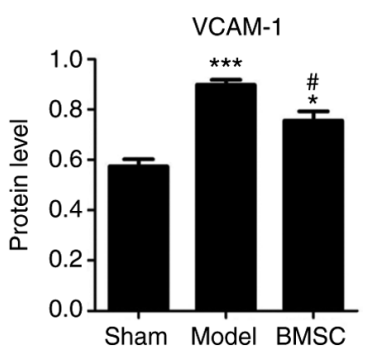

eNOS

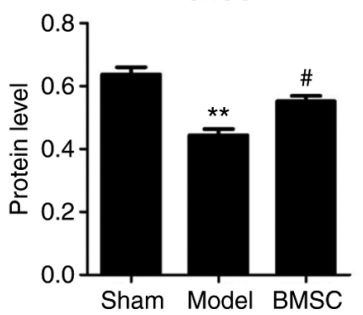

Figure 3. BMSCs suppress endotheliocyte injury in the aortas of OS rats. (A) mRNA expression levels of ET-1, VCAM-1 and eNOS in aortas were detected using reverse transcription-quantitative PCR. (B) Protein expression levels of ET-1, VCAM-1 and eNOS in the aortas were detected using western blotting. (C) Semi-quantitative results of western blotting in all groups. ${ }^{*} \mathrm{P}<0.05,{ }^{* *} \mathrm{P}<0.01$ and ${ }^{* * *} \mathrm{P}<0.001$ vs. the sham group; ${ }^{\#} \mathrm{P}<0.05$ and ${ }^{\# \# \#} \mathrm{P}<0.001 \mathrm{vs}$. the model group. BMSC, bone marrow mesenchymal stem cells; ET-1, endothelin-1; VCAM-1, vascular cell adhesion molecule-1; eNOS, endothelial nitric oxide synthase.

A

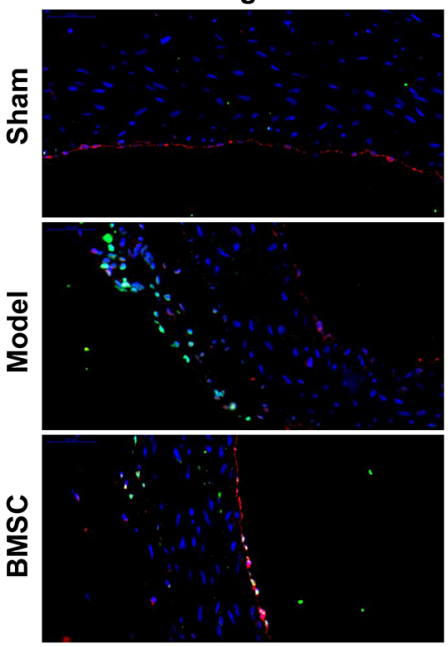

B

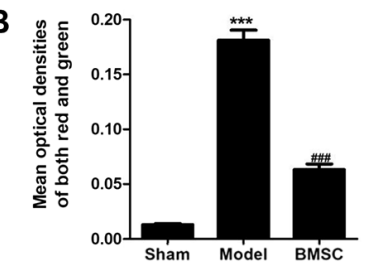

CD34
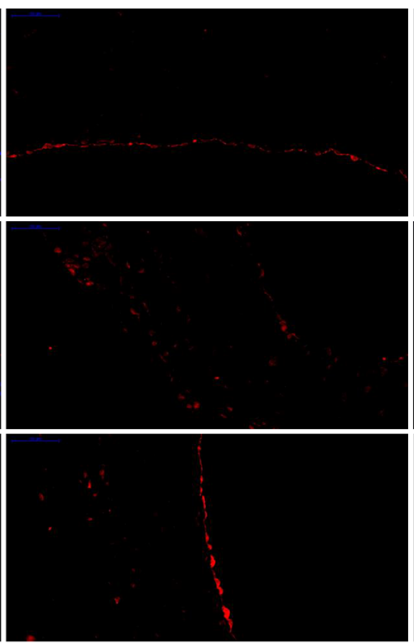

$\mathbf{x} 400$
TUNEL

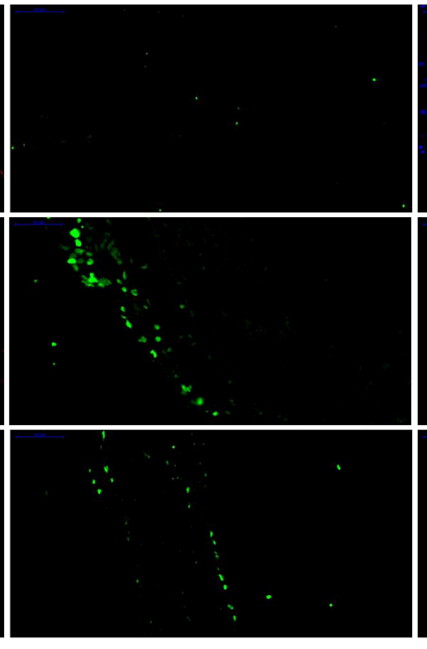

DAPI

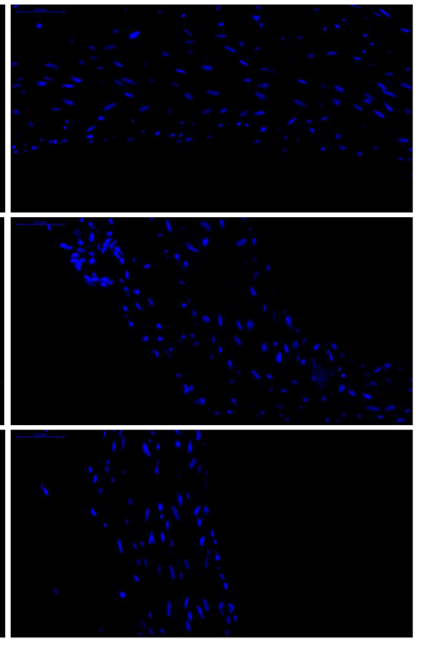

Figure 4. BMSCs suppress endotheliocyte apoptosis in the aortas of OS rats. (A) Apoptosis of endotheliocytes in the aortas of the sham group, model group and BMSC group were detected via TUNEL assays. Green fluorescence indicates apoptotic cells using a TUNEL assay. Red fluorescence indicates the endotheliocytes using a CD34 immunofluorescence assay. Magnification, $\mathrm{x} 400$. The nucleus was stained with DAPI. (B) Quantitative analysis of the co-expression

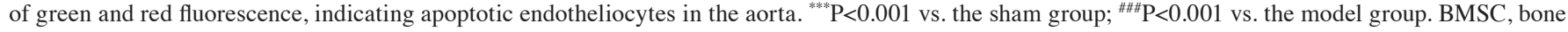
marrow mesenchymal stem cells.

are presented in Fig. 5B. CD31 protein expression was also detected using western blot analysis (Fig. 5C and D), and the same changes were identified as those indicated by the immunohistochemical assay results. Collectively, it was suggested that BMSCs inhibited the apoptosis of endotheliocytes in the aortic tissues of OS rats.

\section{Discussion}

Clinically, OSA combined with COPD is known as OS (30). Both COPD and OSA involve vascular endothelial injury, and the endothelial dysfunction in patients with OS is more serious compared with that of either condition alone and is 


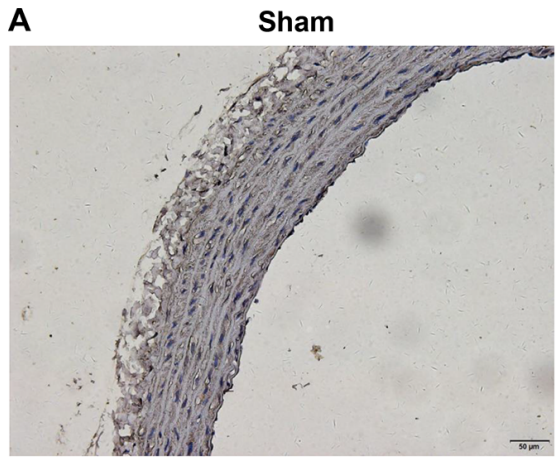

B

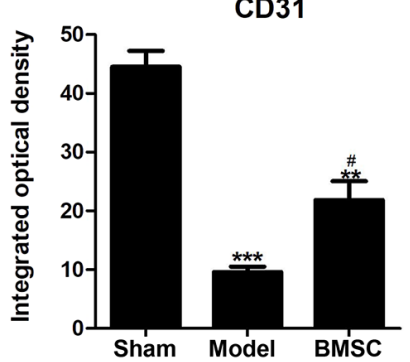

Model

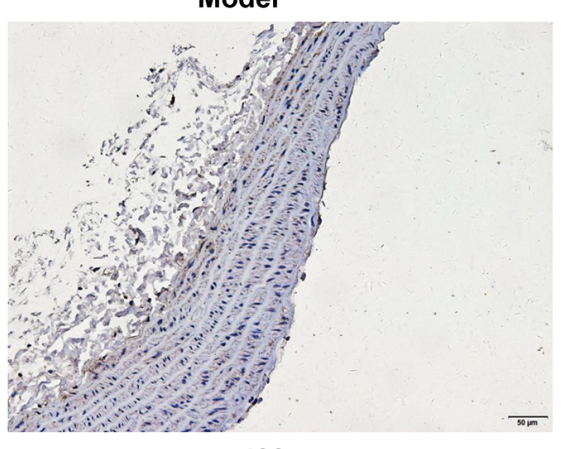

$\times 400$

C

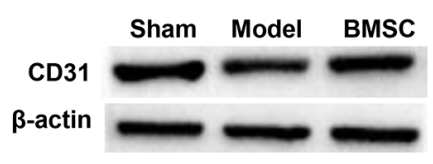

BMSC

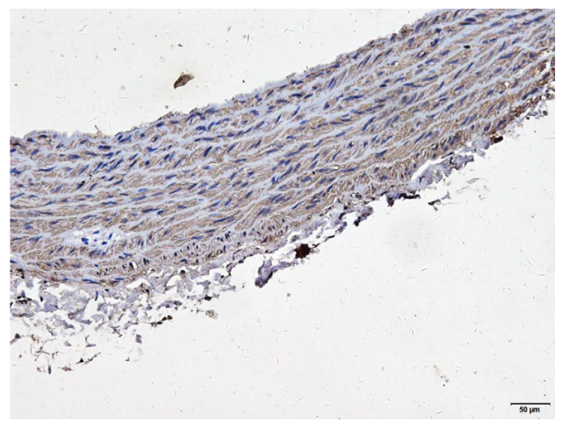

D

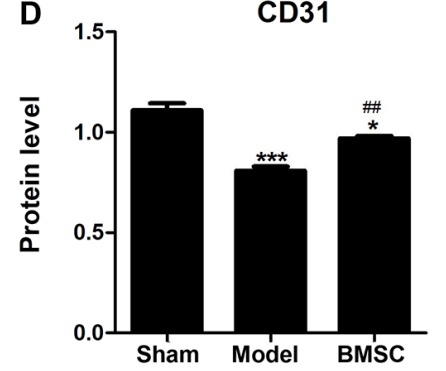

Figure 5. BMSCs suppress the endotheliocyte marker CD31 expression in the aortas of OS rats. (A) Expression of CD31 was detected using an immunohistochemical assay. Magnification, x400. Scale bar, $50 \mu \mathrm{m}$. (B) Quantitative results of the immunohistochemical assay. (C) Protein expression of CD31 in the aortas was detected using western blotting. (D) Semi-quantitative results of western blotting in all groups. ${ }^{*} \mathrm{P}<0.05,{ }^{* *} \mathrm{P}<0.01$ and ${ }^{* * * *} \mathrm{P}<0.001$ vs. the sham group; ${ }^{\#} \mathrm{P}<0.05$ and ${ }^{\# \#} \mathrm{P}<0.01$ vs. the model group. BMSC, bone marrow mesenchymal stem cells.

correlated with the severity of the disease (9). COPD and OSA have different pathogeneses, leading to contradictory clinical treatments of patients with OS (7). Currently, there are no effective drugs for the treatment of OS in clinical practice (14). Therefore, novel therapeutic measures to improve vascular endothelial injury and dysfunction are urgently required.

Cell therapy has been widely used in the study of pulmonary diseases, and its research topics include the treatment of chronic pulmonary diseases and vascular-related complications via cell lung transplantation and circulating stem/progenitor cells (15). BMSCs are non-hematopoietic stem cells with multidifferentiation potential and have the ability to induce tissue regeneration, anti-inflammatory effects and immunosuppression (31). MSC transplantation can downregulate inflammatory mediators in COPD and OSAHS animal models $(32,33)$. It has also been reported that embryonic stem cells and BM-derived EPCs can differentiate into VECs (16). Studies on various animal disease models have revealed that MSCs can repair vascular endothelial injury and correct dysfunction by inhibiting inflammation, oxidative stress and endotheliocyte apoptosis, as well as directly differentiating into endotheliocytes (34-36). Moreover, MSCs alleviate acute lung injury by enhancing anti-inflammatory pathways (37). Based on the aforementioned studies, it was hypothesized that BMSC therapy may be a new and effective treatment approach for vascular endothelial injury, dysfunction or apoptosis in patients with OS. To the best of our knowledge, few studies have investigated the effect of BMSCs on aortic endothelial injury in an OS rat model.

Our previous experiment demonstrated that the inhibition of the progression of emphysema by BMSCs in the OS model may be via the differentiation of BMSCs into endotheliocytes, consequently suppressing endotheliocyte apoptosis, and by their antioxidative stress function (27). However, the effect of BMSC transplantation on aortic injury remained unknown. The present study constructed an OS rat model using cigarette smoke and intermittent hypoxia. It was found that transplanted BMSCs migrated not only into lung tissues, but also into the aorta, heart, liver, spleen, kidney and brain. However, the greatest number of BMSCs migrated to lung and aortic tissues. Granulocyte colony-stimulating factor can promote the migration of BMSCs to damaged lung tissue, ultimately alleviating pulmonary fibrosis (38). Extracellular vesicles derived from BMSCs could be beneficial in undertaking a reparative effort in abdominal aortic aneurysm-induced degeneration of vascular tissue (39). BMSCs can migrate to damaged tissues $(40,41)$, and in the current study, BMSCs are present not only in the lungs but also in other tissues. This result indicates that the OS model can lead to severe aortic and lung injury. The present study detected aortic injury using $\mathrm{H} \& \mathrm{E}$ and Masson staining. H\&E staining demonstrated that the vascular wall structure of the aorta was disordered, and the boundary between the outer membrane, middle membrane and intima was not clear. The elastic membrane of the media was not visible, smooth muscle cells were disordered as well as showed swelling, hyperplasia and cell morphology changes, and endothelial swelling was observed in the OS groups. Moreover, the expression of collagen fibers in the outer and middle aortic membranes of the OS group was significantly increased, and its elastic fibers were markedly decreased. However, in the BMSC group, BMSCs alleviated vascular injury and suppressed the formation of collagen fibers. These results suggested that arterial tissues were severely damaged in the OS model and that BMSCs can inhibit these damages.

Nitric oxide (NO) and ET-1 are antagonistic vascular active substances synthesized and are secreted by VECs $(42,43)$. ET-1 negatively regulates the release of NO in the endothelium, and the increase in NO concentration inhibits the formation 
of ET-1. The dynamic balance between the two maintains normal vascular endothelial function, and an increase of ET-1 and a decrease of NO are markers of vascular endothelial injury $(44,45)$. eNOS can synthesize NO. In previous studies, numerous factors in vivo and in vitro were revealed to accelerate or delay the process of reendothelialization by upregulating or downregulating the expression of eNOS $(46,47)$. It has been reported that VCAM-1 is highly expressed in rat aortic endothelial cells after balloon injury, and the expression of VCAM-1 enhances with increasing inflammatory response time (48). From the present RT-qPCR and western blotting results, the expression levels of ET-1 and VCAM-1 were found to be significantly promoted, while the expression of eNOS was significantly inhibited in OS rats compared with the sham rats. Furthermore, an immunohistochemical assay was used to analyze the expression of CD31 in the injured vessels. It was identified that BMSCs could suppress endothelial cell apoptosis. Thus, the current study indicated that BMSCs may be potent therapeutic tools for vascular injury caused by OS.

There are some limitations to the present study. It was identified that BMSCs were mainly localized to the lung and aortic tissues, and a protective effect of BMSCs on aortic injury of OS model was observed. However, the related mechanism via which BMSCs protected against aortic injury remains unknown.

In conclusion, the present study demonstrated the therapeutic effect of BMSC administration for aortic injury in OS rats induced by cigarette smoke and intermittent hypoxia. It was identified that BMSCs inhibited aortic injury in the OS model by suppressing endotheliocyte apoptosis. However, the detailed mechanism among these changes requires further investigation.

\section{Acknowledgements}

Not applicable.

\section{Funding}

This work was supported by the National Natural Science Foundation of China (grant no. 81560010) and the Science and Technology Planning Project of Yunnan Province [grant no. 2017FE467(-100)].

\section{Availability of data and materials}

The datasets used and/or analyzed during the current study are available from the corresponding author on reasonable request.

\section{Authors' contributions}

ZJ, HB, JH, XH and JD conceived and designed the study. MC, ZH, CY, LY, LH, HL, KZ and QW performed the experiments. HL, KZ and QW processed the data. LH, HB, JH, XH and JD wrote the manuscript. $\mathrm{HB}, \mathrm{JH}$ and $\mathrm{ZJ}$ reviewed and edited the manuscript. All authors read and approved the final manuscript.

\section{Ethics approval and consent to participate}

The animal experiments were approved by the Animals Ethics Committee of The First People's Hospital of Kunming and in accordance with the Guide for the Care and Use of Laboratory Animals.

\section{Patient consent for publication}

Not applicable.

\section{Competing interests}

The authors declare that they have no competing interests.

\section{References}

1. Tsai SC: Chronic obstructive pulmonary disease and sleep related disorders. Curr Opin Pulm Med 23: 124-128, 2017.

2. Portegies ML, Lahousse L, Joos GF, Hofman A, Koudstaal PJ, Stricker BH, Brusselle GG and Ikram MA: Chronic obstructive pulmonary disease and the risk of stroke. The Rotterdam Study. Am J Respir Crit Care Med 193: 251-258, 2016.

3. Gottlieb DJ and Punjabi NM: Diagnosis and management of obstructive sleep Apnea: A review. JAMA 323: 1389-1400, 2020.

4. Shahar E, Whitney CW, Redline S, Lee ET, Newman AB, Nieto FJ, O'Connor GT, Boland LL, Schwartz JE and Samet JM: Sleep-disordered breathing and cardiovascular disease: Cross-sectional results of the Sleep Heart Health Study. Am J Respir Crit Care Med 163: 19-25, 2001.

5. Munoz R, Duran-Cantolla J, Martínez-Vila E, Gallego J, Rubio R, Aizpuru F, De La Torre G: Severe sleep apnea and risk of ischemic stroke in the elderly. Stroke 37: 2317-2321, 2006.

6. Flenley DC: Sleep in chronic obstructive lung disease. Clin Chest Med 6: 651-661, 1985.

7. Marin JM, Soriano JB, Carrizo SJ, Boldova A and Celli BR: Outcomes in patients with chronic obstructive pulmonary disease and obstructive sleep apnea: The overlap syndrome. Am J Respir Crit Care Med 182: 325-331, 2010.

8. Sharma B, Neilan TG, Kwong RY, Mandry D, Owens RL, McSharry D, Bakker JP and Malhotra A: Evaluation of right ventricular remodeling using cardiac magnetic resonance imaging in co-existent chronic obstructive pulmonary disease and obstructive sleep apnea. COPD 10: 4-10, 2013.

9. Thomashow MA, Shimbo D, Parikh MA, Hoffman EA, Vogel-Claussen J, Hueper K, Fu J, Liu CY, Bluemke DA, Ventetuolo CE, et al: Endothelial microparticles in mild chronic obstructive pulmonary disease and emphysema. The Multi-Ethnic Study of Atherosclerosis Chronic Obstructive Pulmonary Disease study. Am J Respir Crit Care Med 188: 60-68, 2013.

10. McNicholas WT: COPD-OSA overlap syndrome: Evolving evidence regarding epidemiology, clinical consequences, and management. Chest 152: 1318-1326, 2017.

11. Willerson JT and Ridker PM: Inflammation as a cardiovascular risk factor. Circulation 109 (Suppl 1): II2-II10, 2004

12. Greenberg H, Ye X, Wilson D, Htoo AK, Hendersen T and Liu SF: Chronic intermittent hypoxia activates nuclear factor-kappaB in cardiovascular tissues in vivo. Biochem Biophys Res Commun 343: 591-596, 2006.

13. Ryan S and McNicholas WT: Intermittent hypoxia and activation of inflammatory molecular pathways in OSAS. Arch Physiol Biochem 114: 261-266, 2008.

14. Rezaeetalab F, Rezaeitalab F and Dehestani V: Inhaled steroids reduce apnea-hypopnea index in overlap syndrome. Pneumologia 62: 212-214, 2013.

15. Heise RL, Link PA and Farkas L: From here to there, progenitor cells and stem cells are everywhere in lung vascular remodeling. Front Pediatr 4: 80, 2016.

16. Ando J and Yamamoto K: Vascular mechanobiology: Endothelial cell responses to fluid shear stress. Circ J 73: 1983-1992, 2009.

17. Shi Z, Chen Y, Cao J, Zeng H, Yang Y, Chen P, Luo H, Peng H, Cai S and Guan C: Intratracheal transplantation of endothelial progenitor cells attenuates smoking-induced COPD in mice. Int J Chron Obstruct Pulmon Dis 12: 947-960, 2017.

18. Sun YQ, Deng MX, He J, Zeng QX, Wen W, Wong DS, Tse HF, Xu G, Lian Q, Shi J and Fu QL: Human pluripotent stem cell-derived mesenchymal stem cells prevent allergic airway inflammation in mice. Stem Cells 30: 2692-2699, 2012. 
19. Luo D, Yan X, Liu D, Zhou X and Liu G: Differential effects of mesenchymal stem cells on a heterogeneous cell population within lung cancer cell lines. Mol Cell Biochem 378: 107-116, 2013.

20. Le Blanc K, Tammik C, Rosendahl K, Zetterberg E and Ringden O: HLA expression and immunologic properties of differentiated and undifferentiated mesenchymal stem cells. Exp Hematol 31: 890-896, 2003.

21. Allers C, Sierralta WD, Neubauer S, Rivera F, Minguell JJ and Conget PA: Dynamic of distribution of human bone marrow-derived mesenchymal stem cells after transplantation into adult unconditioned mice. Transplantation 78: 503-508, 2004.

22. Carreras A, Almendros I and Farré R: Potential role of bone marrow mesenchymal stem cells in obstructive sleep apnea. Int J Stem Cells 4: 43-49, 2011.

23. Park JS, Kim HK, Kang EY, Cho R and Oh YM: Potential therapeutic strategy in chronic obstructive pulmonary disease using pioglitazone-augmented Wharton's Jelly-derived mesenchymal stem cells. Tuberc Respir Dis (Seoul) 82: 158-165, 2019.

24. Liu HM, Liu YT, Zhang J and Ma LJ: Bone marrow mesenchymal stem cells ameliorate lung injury through anti-inflammatory and antibacterial effect in COPD mice. J Huazhong Univ Sci Technolog Med Sci 37: 496-504, 2017.

25. Shangguan L, Li X, Wang $Z$ and Luo Z: Transforming growth factor- $\beta 1$ induces bone marrow-derived mesenchymal stem cells to differentiate into cancer-associated fibroblasts. Zhonghua Zhong Liu Za Zhi 37: 804-809, 2015 (In Chinese).

26. Ding W, Li J, Singh J, Alif R, Vazquez-Padron RI, Gomes SA, Hare JM and Shehadeh LA: miR-30e targets IGF2-regulated osteogenesis in bone marrow-derived mesenchymal stem cells, aortic smooth muscle cells, and $\mathrm{ApoE}^{-/-}$mice. Cardiovasc Res 106: 131-142, 2015.

27. Chen M, Huang Z, Bi H, Pan X, He J, He L, He X, Du J, Zhou K, Wang L, et al: Effects of bone marrowderived mesenchymal stem cell transplantation on chronic obstructive pulmonary disease/obstructive sleep apnea overlap syndrome in rats. Mol Med Rep 20: 4665-4673, 2019

28. Tang XJ, Wang B, Huang PY, Guo ZT, Tang QL, Li SS and Yang XM: Effects of chronic intermittent hypoxia on blood pressure and vascular remodeling. Zhonghua Er Bi Yan Hou Tou Jing Wai Ke Za Zhi 54: 601-605, 2019 (In Chinese).

29. Livak KJ and Schmittgen TD: Analysis of relative gene expression data using real-time quantitative PCR and the 2(-Delta Delta C(T)) method. Methods 25: 402-408, 2001.

30. Zhang XW, Cai W, Jin F, Zhang YQ and Zhang XL: Effect of Bi-level positive airway pressure ventilator on the heart function and vascular endothelial function of patients with the overlap syndrome. Zhonghua Jie He He Hu Xi Za Zhi 34: 17-20, 2011 (In Chinese).

31. Kadota T, Fujita Y, Yoshioka Y, Araya J, Kuwano K and Ochiya T: Extracellular vesicles in chronic obstructive pulmonary disease. Int J Mol Sci 17: 1801, 2016.

32. Weiss DJ, Casaburi R, Flannery R, LeRoux-Williams M and Tashkin DP: A placebo-controlled, randomized trial of mesenchymal stem cells in COPD. Chest 143: 1590-1598, 2013.

33. Carreras A, Almendros I, Montserrat JM, Navajas D and Farré R: Mesenchymal stem cells reduce inflammation in a rat model of obstructive sleep apnea. Respir Physiol Neurobiol 172: 210-212, 2010.

34. Luan Y, Zhang ZH, Wei DE, Zhao JJ, Kong F, Cheng GH and Wang YB: Implantation of mesenchymal stem cells improves right ventricular impairments caused by experimental pulmonary hypertension. Am J Med Sci 343: 402-406, 2012.
35. Calió ML, Marinho DS, Ko GM, Ribeiro RR, Carbonel AF, Oyama LM, Ormanji M, Guirao TP, Calió PL, Reis LA, et al: Transplantation of bone marrow mesenchymal stem cells decreases oxidative stress, apoptosis, and hippocampal damage in brain of a spontaneous stroke model. Free Radic Biol Med 70: 141-154, 2014.

36. Hung SC, Pochampally RR, Chen SC, Hsu SC and Prockop DJ: Angiogenic effects of human multipotent stromal cell conditioned medium activate the PI3K-Akt pathway in hypoxic endothelial cells to inhibit apoptosis, increase survival, and stimulate angiogenesis. Stem Cells 25: 2363-2370, 2007.

37. Luo F, Jiang W, Xu Y, Liu XM, Wang W, Zhang W and Luo C: The mechanisms involved in mesenchymal stem cell alleviation of sepsis-induced acute lung injury in mice: A pilot study. Curr Ther Res Clin Exp 93: 100593, 2020.

38. Zhao FY, Cheng TY, Yang L, Huang YH, Li C, Han JZ, Li XH, Fang LJ, Feng DD, Tang YT, et al: G-CSF inhibits pulmonary fibrosis by promoting BMSC homing to the lungs via SDF-1/CXCR4 chemotaxis. Sci Rep 10: 10515, 2020

39. Sajeesh S, Broekelman T, Mecham R and Ramamurthi A: Stem cell derived extracellular vesicles for vascular elastic matrix regenerative repair. Acta Biomate 113: 267-278, 2020.

40. Yamada M, Kubo H, Kobayashi S, Ishizawa K, Numasaki M, Ueda S, Suzuki T and Sasaki H: Bone marrow-derived progenitor cells are important for lung repair after lipopolysaccharide-induced lung injury. J Immunol 172: 1266-1272, 2004.

41. Ortiz LA, Gambelli F, McBride C, Gaupp D, Baddoo M, Kaminski N and Phinney DG: Mesenchymal stem cell engraftment in lung is enhanced in response to bleomycin exposure and ameliorates its fibrotic effects. Proc Natl Acad Sci USA 100: 8407-8411, 2003.

42. Konczalla J, Wanderer S, Mrosek J, Schuss P, Platz J, Güresir E, Seifert V and Vatter H: Crosstalk between the angiotensin and endothelin-system in the cerebrovasculature. Curr Neurovasc Res 10: 335-345, 2013.

43. Millatt LJ, Abdel-Rahman EM and Siragy HM: Angiotensin II and nitric oxide: A question of balance. Regul Pept 81: 1-10, 1999.

44. Signorello MG, Viviani GL, Armani U, Cerone R, Minniti G, Piana A and Leoncini G: Homocysteine, reactive oxygen species and nitric oxide in type 2 diabetes mellitus. Thromb Res 120: 607-613, 2007.

45. Wiwanitkit V: Endothelin-1 and protein kinase C co-expression in the pathogenesis of diabetic retinopathy. J Diabetes Complications 21: 359-362, 2007.

46. Urao N, Okigaki M, Yamada H, Aadachi Y, Matsuno K, Matsui A, Matsunaga S, Tateishi K, Nomura T, Takahashi T, et al: Erythropoietin-mobilized endothelial progenitors enhance reendothelialization via Akt-endothelial nitric oxide synthase activation and prevent neointimal hyperplasia. Circ Res 98: 1405-1413, 2006.

47. Schwartz R, Osborne-Lawrence S, Hahner L, Gibson LL, Gormley AK, Vongpatanasin W, Zhu W, Word RA, Seetharam D, Black S, et al: C-reactive protein downregulates endothelial NO synthase and attenuates reendothelialization in vivo in mice. Circ Res 100: 1452-1459, 2007

48. Tanaka H, Sukhova GK, Swanson SJ, Clinton SK, Ganz P, Cybulsky MI and Libby P: Sustained activation of vascular cells and leukocytes in the rabbit aorta after balloon injury. Circulation 88: 1788-1803, 1993.

This work is licensed under a Creative Commons Attribution-NonCommercial-NoDerivatives 4.0 International (CC BY-NC-ND 4.0) License. 